\title{
Association cortex lesions and stimulus generalization in cats*
}

\author{
LOWELL W. COUTANT \\ Wheaton College, Norton, Massachusetts 02766
}

\begin{abstract}
Following go/no-go discrimination training, cats with large anterior or posterior association cortex lesions were tested for excitatory and inhibitory generalization of response to line tilt. Frontal Ss were slightly, but insignificantly, impaired in learning the go/no-go discrimination relative to the posterior and control Ss. There were no differences between lesion groups in form or level of generalization gradients. Generalization decrements following excitatory training were much more pronounced than those following inhibitory training, although there was some evidence of inhibitory control.
\end{abstract}

According to Brutkowski $(1964,1965)$, anterior cortical injuries produce a severe form of disinhibition in mammals which is manifested as an abnormal difficulty in suppressing positive responses to the negative stimulus in differentiation conditioning and go/no-go discrimination. Frontal ablations impair go/no-go discrimination learning by dogs and monkeys (Battig, Rosvold, \& Mishkin, 1962). The experiment described below was carried out to determine whether frontal lesions would also impair go/no-go discrimination learning in cats.

It was also intended to investigate the effects of frontal damage on stimulus generalization. Brutkowski's (1965) hypothesis does not make specific predictions regarding generalization phenomena; nevertheless, it seems reasonable to predict that disinhibitory effects would increase overall level of response strength, decrease the slope of the generalization gradient, or both.

Inhibitory stimulus control has been demonstrated by Honig, Boneau, Burstein, and Pennypacker (1963). Pigeons trained successively on a discrimination between a lighted disk intersected by a vertical black line and a similar disk with no line generated roughly similar excitatory and inhibitory generalization gradients. That is, birds trained with the line stimulus positive and plain disk negative produced typical bell-shaped generalization curves when the angle of the line was varied during generalization testing. Birds trained with the line stimulus negative and the plain disk positive generated curves that were approximately inverse to those above. Thus, their experiment demonstrated clear gradients of generalization of both excitation and inhibition, and provides an attractive paradigm for functional analysis of anterior cortex in cats.

The experiment described below was thus intended to

*This research was supported by Grant MH 4726 from the National Institute of Mental Health, U.S. Public Health Service, to J. M. Warren. Part of these data were submitted to the Graduate School of The Pennsylvania State University in partial fulfillment of the requirements for the $\mathrm{PhD}$ degree. Special thanks are due Professor Warren. whose advice contributed to this experiment from inception to conclusion. investigate whether: (a) frontal lesions in cats would produce impairment in go/no-go discrimination, (b) these lesions would affect stimulus generalization, and (c) inhibitory stimulus control could be demonstrated in cats as well as in pigeons.

\section{METHOD}

The Ss were 24 cats, approximately 1 year old at time of surgery, all with very similar experience in two previous experiments involving simultaneous visual discrimination (Mumma \& Warren, 1968; Derdzinski, 1967). Three groups of eight cats were matched on the basis of performance in a simultaneous discrimination in the Wisconsin General Testing Apparatus (WGTA). Each $S$ then received one of the following lesions:

Anterior. Complete aspiration of the cortex of the proreal gyri and the lateral banks of the presylvian sulci (the frontal "granular" cortex of A kert, 1964), the anterior sigmoid gyri, and the anterior $2-3 \mathrm{~mm}$ of the posterior sigmoid gyri, extending laterally to the depths of the coronal sulci (the pericruciate association area of Thompson, Johnson, and Hoopes, 1963). These removals included the depths of the cruciate and presylvian sulci and the cortex of the medial walls of these areas. Previous lesions of gyrus proreus have been relatively limited in ventral extent by the difficulty of visualizing produced by surrounding bone. To increase the extent of these removals, the posteromedial wall of the orbital fossa was removed to the level of the olfactory tract.

Posterior. Complete removal of the middle and posterior suprasylvian gyri to the depths of adjacent sulci, with one exception: the dorso-posterior "corner" of this area was spared so as to minimize the possibility of undercutting projections to the primary visual cortex.

Control. Since it is conceivable that the extensive removals of bone and retraction necessitated by the approach to the anterior cortex described above could produce lasting defects in visual convergence, two sham-operated controls were subjected to removal of bone identical to that of the anterior Ss. The dura was not incised in these preparations. In six other controls, similar removals of bone were made, but the posterior wall of the frontal sinus was not disturbed. That is, bone adjacent to the cortical surface was not removed in these Ss. It was assumed that posterior bone damage alone would not produce changes in the behavior described below.

All testing was conducted in a WGTA. Two $7.6-\mathrm{cm}$ vertical white disks were presented successively on a gray formboard with one centered foodwell. One of these disks was bisected by a 9-mm black line. continuously variable with respect to angle. The second disk was plain. 


\section{Procedure}

Within each of the three lesion groups, Ss were placed in two matched subgroups of four individuals. One of these subgroups was trained with the movable line stimulus positive and the plain stimulus negative; for the second subgroup, the converse was true. Throughout training, the movable line stimulus was set in its vertical (90-deg) position. Ss were presented successively with the two stimuli for 50 trials each day until each $\mathrm{S}$ reached a criterion of 18 correct responses in 20 consecutive trials. That is, only one stimulus was presented on each trial, the order of presentation being regulated by a Gellermann series. A correct response was defined as one in which $S$ displaced the positive stimulus within $10 \mathrm{sec}$ or withheld response to the negative stimulus for more than $10 \mathrm{sec}$. S was rewarded with a $7-\mathrm{mm}$ cube of raw pork kidney on displacing the positive stimulus. A trial was terminated either when S displaced the stimulus or when $10 \mathrm{sec}$ elapsed. After reaching criterion, Ss were tested for generalization.

Generalization tests were presented according to a balanced square, which varied the presentation order of seven stimulus line angles $(0,30,60,90,120,150$, and $180 \mathrm{deg})$ over 7 test days. On each generalization test day, $S$ received an initial 10 trials of go/no-go discrimination training with differential reward and with the movable line set at $90 \mathrm{deg}$, as described above. S was then presented with five test trials with the movable line stimulus set at one of the above angles. Only the movable line stimulus was presented on these trials; it was not rewarded. S was then given six discrimination trials, followed by five generalization test trials with the line set at a different angle, six discrimination trials, five test trials, and so on, until the sequence of seven line angles for a given day was completed. Thus $\mathrm{S}$ was presented each day with a total of 46 discrimination trials, 23 of which were rewarded, and 35 test trials, none of which was rewarded. Response latency was recorded on each test trial with a Standard .01-sec timer controlled by a footswitch and was limited to $10 \mathrm{sec}$.

\section{RESULTS}

Reconstructions of cortical lesions and thalamic degeneration based on photographs taken during sectioning and on thionin-stained frozen sections may be viewed elsewhere (Coutant, 1968). Most frontal lesions were quite complete and produced minimal direct damage to subcortical structures. Four Ss showed evidence of primary or secondary damage to small areas of the caudate nucleus and/or the putamen. One of these incurred small, asymmetrical, bilateral caudate lesions; in the other three, damage was unilateral. All frontal Ss sustained considerable damage to subcortical white matter, most of it projecting to the cortical areas ablated. In general, degeneration of nucleus medialis dorsalis (MD) was less extensive in these animals than Akert's (1964) description of degeneration following complete aspiration of gyrus proreus and the banks of the presylvian sulcus. In no case was MD completely degenerated: in most of the animals, degeneration was complete only in limited areas in the center of the nucleus. Similarly, degeneration in the basolateral nuclei was relatively sparse and limited to the more medial portions of these nuclei.

Reconstructions of the lesions of two animals in the posterior group were not made due to an error in photography. The six animals with reconstructed posterior lesions sustained relatively limited damage. Most lesions were limited to superficial surface areas of middle suprasylvian gyrus with little tissue removed from the banks of the gyrus. Damage to posterior suprasylvian areas was somewhat more complete. One posterior $\mathrm{S}$ sustained severe bilateral undercutting of visual radiations and nearly complete degeneration of the lateral geniculate nucleus (LGN) and is not included in the following data. A second animal sustained moderate bilateral degeneration of LGN, and two others showed minimal, unilateral LGN degeneration. In the remaining four posterior Ss, there was no observable degeneration in LGN and retrograde changes in pulvinar were limited generally to the lateral ventral half of the nucleus.

\section{Go/No-Go Discrimination}

Five of eight frontals, three of seven posteriors, and two of eight controls failed the go/no-go discrimination after 1,500 trials. These proportions do not differ significantly (Fisher's exact probability test, $\mathrm{p}>.05$ ). Neither were there any significant differences in errors or trials to criterion on this task. Trials to criterion for the successful Ss were as follows: frontals, 494, 547, and 1,473; posteriors, $243,1,170,1,323$, and 1,344: normals, 386, 471, 570, 870, 1,070, and 1,101. Note that two frontals learned in fewer trials than the median normal or posterior cases. The slight trend of the frontal cats toward an impairment in go/no-go discrimination was not due to a differential tendency to respond positively on "no-go" trials; they made more errors on "go" trials as well. (Median errors on "go" trials were 7.5, 3.0, and 2.5 for frontals, posteriors, and controls: median errors on "no-go" trials were 656,601 , and 421 for the same three groups.)

\section{Stimulus Generalization}

Performance on discrimination training trials interspersed among generalization test trials remained at a high level throughout, averaging $83 \%$ correct for all animals over the 7 days of generalization testing. Median response times for all 7 days of generalization testing are plotted over stimulus line angle in Fig. 1 for the successful animals in each of the three lesion groups. "Line -" indicates generalization following discrimination training with the plain disk rewarded and the movable line stimulus nonrewarded; "Line +" indicates generalization following training with the movable line stimulus rewarded. There were no significant differences between the three lesion groups under either reward condition.

The data for the normal and brain-injured cats are accordingly combined in Fig. 2 . The excitatory gradient is very similar to those obtained from pigeons tested under similar circumstances (Guttman \& Kalish, 1956; Honig et al, 1963). The gradient of inhibition is, 
however, extremely broad, showing little apparent generalization decrement over the range of values tested, although the decrease in latencies from 30 to $0 \mathrm{deg}$ and from 150 to $180 \mathrm{deg}$ is highly significant; the latency for none of the cats was shorter on the trials with 30 - and 150-deg tilts than with 0 or $180 \mathrm{deg}$.

\section{DISCUSSION}

Brutkowski's disinhibition theory predicts that frontal lesions will severely impair the suppression of responses on negative trials that is required for solution of go/no-go discriminations by mammals. In the present group, some cats with very extensive frontal damage were quite capable of suppressing such responses and, in fact, sometimes withheld responses on positive trials. This finding is clearly incompatible with Brutkowski's theory. It is, however, consistent with the suggestion of Warren, Coutant, and Cornwell (1969) that "inhibition" and the "frontal deficit" in cats cannot be considered as unitary phenomena.

In their experiments, cats with frontal lesions were tested on a variety of tasks involving "inhibition." They found little correspondence between the results on these
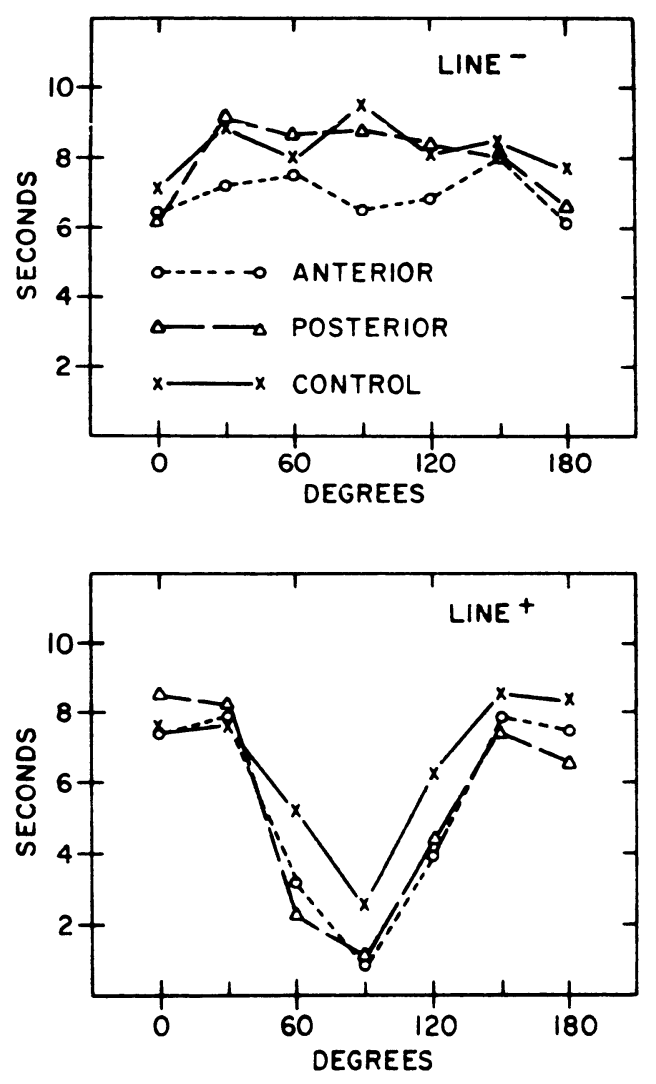

Fig. 1. Median response times vs stimulus line angle. "Line -" and "Line +" indicate whether plain disk or movable line stimulus was rewarded in discriminating training. The movable line was set at 90 deg throughout training.

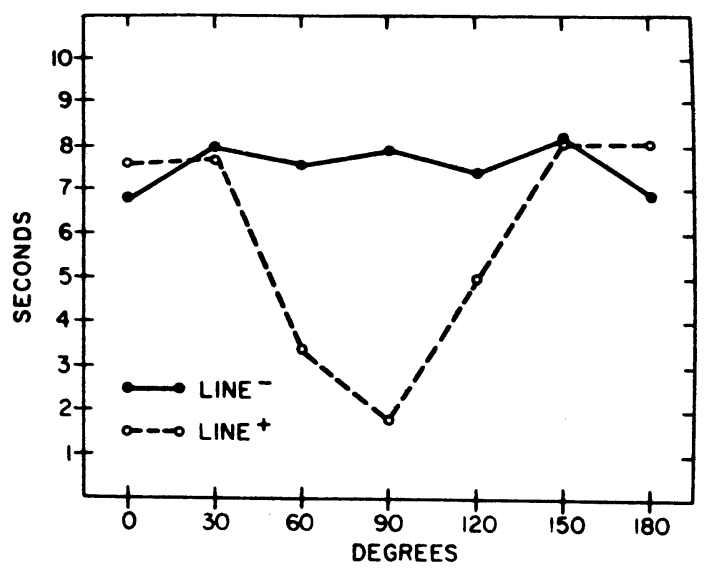

Fig. 2. Mean response times vs line angle. "Line -" includes data from 2 anteriors, 2 posteriors, and 3 controls; "Line +" includes data from 1 anterior, 2 posteriors, and 3 controls.

tasks. For example, cats that were severely impaired in extinguishing a running response following overtraining were much less disrupted in extinguishing a reaching response in the WGTA and in learning a go/no-go discrimination between stimuli much more disparate than those used in the present experiment. Brutkowski's treatment of frontal cortex as part of a general inhibitory system is clearly an oversimplification and needs much more thorough analysis.

This suggestion is supported by the observation that the cats with anterior and posterior association cortex lesions did not differ in generalization behavior.

The overall results of the generalization tests are obviously in very good agreement with those previously obtained in operant experiments with pigeons in respect to the gradient of excitation. The gradient of inhibition obtained from the cats in this study is extremely broad and shows less generalization decrement than is frequently seen in pigeons. It is important, therefore, to reiterate that the decrease in latencies observed on the extreme (0- and 180-deg) points is highly significant when tested against the average latencies at the adjacent (30- and 150-deg) points. Although this generalization decrement is not visually impressive, it is highly significant statistically and indicates that line tilt acquired stimulus control over both excitation and inhibition. It will be recalled that Honig et al found that with continued testing, negative gradients became flatter relative to the positive gradients in pigeons. Thus, there is a fairly substantial degree of correspondence between the results from cats and pigeons. The argument between the present findings and those of Honig et al may be regarded as impressive in view of the difference in species, apparatus (WGTA vs Skinner box), training procedures, and responses (free vs restricted operants). Indeed, one may argue that the primary value of this experiment lies in its demonstration that the techniques developed for the analysis of stimulus control in operant 
experiments with birds may be successfully modified to investigate similar problems with mammals in the WGTA.

\section{REFERENCES}

Akert. K. Comparative anatomy of the frontal cortex and thalemocortical connections. In J. M. Warren and K. Akert (Eds.). The frontal gramular cortex and behavior. New York: McGraw-Hill. 1964. Pp. 372-396.

Battig, K.. Rosvold, H. E.. \& Mishkin. M. Comparison of the effects of frontal and caudate lesions on discrimination learning in monkey's. Journal of Comparative \& Physiological Psychology, 1962, 55, 458-463.

Brutkowski, S. Prefrontal cortex and drive inhibition. In J. M. Warren and $\mathrm{K}$. Akert (Eds.). The frontal granular cortex and behavior. New York: McGraw-Hill, 1964. Pp. 242-270.

Brutkowski. S. Functions of prefrontal cortex in animals. Physiological Review, 1965, 45, 721-746.

Coutant. L. W. Association cortex lesions and behavior in cats. (Doctoral dissertation, Pennsylvania State University) Ann Arbor. Mich: University Microfilms. 1968. No. 69-5538.
Derdzinski. D. Shape discrimination learning by cats. Unpublished master's thesis. Pennsylvania State University. 1967.

Guttman. N.. \& Kalish. H. I. Discriminability and stimulus generalization. Journal of Experimental Psychology. 1956. 51. 79-88.

Honig, W. K.. Boneau, C. A.. Burstein. K. R.. \& Pennypacker. H. $S$. Positive and negative generalization gradients obtained after equivalent training conditions. Journal of Comparative \& Physiological Psychology. 1963. 56, 111-116.

Mumma, R., \& Warren, J. M. Two-cue discrimination learning by cats. Journal of Comparative \& Physiological Psychology: 1968. 66, 116-121.

Thompson. R. F., Johnson, R. H.. \& Hoopes, J. J. Organization of auditory. somatic sensory and visual projection to association fields of cerebral cortex in the cat. Journal of Neurophysiology, 1963, 26, 343-364.

Warren. J. M., Coutant, L. W.. \& Cornwell. P. R. Cortical lesions and response inhibition in cats. Neuropsychologia, 1969. 7. 245-247.

(Received for publication August 21, 1972: revision received January $29,1973$. 